\title{
Oximetría de pulso y su eficacia para diagnosticar vitalidad pulpar.
}

\author{
Pulse oximetry and its use in diagnosing pulp vitality.
}

\author{
Magdalena de la Rosa-Reyes, ${ }^{*}$ Luis Alejandro Aguilera-Galaviz, ${ }^{\ddagger}$ Roxana Araujo-Espino, ${ }^{\S}$ \\ Óscar Cepeda-Argüelles, ${ }^{\ddagger}$ César Gaitán-Fonseca, ${ }^{\ddagger}$ Carlos Bermúdez-Jiménez ${ }^{\ddagger}$
}

\section{RESUMEN}

Objetivo: Identificar y analizar en la literatura científica reciente el uso de la oximetría de pulso como método diagnóstico de vitalidad pulpar. Metodología: En este trabajo se llevó a cabo una revisión de la literatura en las plataformas de PubMed y Google Académico, en la cual se analizó el uso de la oximetría de pulso en el diagnóstico y monitoreo odontológico del estado pulpar. Resultados: Después de una exhaustiva revisión, y de acuerdo con los criterios de inclusión y exclusión, se analizaron 21 artículos. La mayoría de los trabajos consideran la oximetría de pulso un método alternativo de diagnóstico indoloro, seguro y eficaz; sin embargo, la adaptación de un instrumento de uso exclusivo odontológico es necesaria para una medición exacta de la saturación de oxígeno en la pulpa dental. Los avances tecnológicos en el campo clínico de la odontología nos han llevado a la búsqueda de nuevas técnicas diagnósticas clínicas para mejorar la atención y los tratamientos de los pacientes que acuden día con día a recibir una consulta odontológica. Conclusiones: En los últimos años la oximetría de pulso ha demostrado ser una herramienta de diagnóstico eficaz para el diagnóstico de la vitalidad pulpar. El análisis de los artículos incluidos en esta revisión concluye que la oximetría de pulso es una técnica innovadora que puede ser utilizada como una herramienta diagnóstica adyuvante en el diagnóstico de la vitalidad pulpar.

Palabras clave: Oximetría, cavidad pulpar, pulpitis, diagnóstico, prueba de la pulpa dental.

\section{ABSTRACT}

Objective: To identify and analyze in the recent scientific literature the use of pulse oximetry as a diagnostic method for pulp vitality. Methodology: In this work, a literature review was carried out on the PubMed and Google Scholar platforms in which the use of pulse oximetry in the dental diagnosis and monitoring of pulp status was analyzed. Results: After an exhaustive review and in accordance with the inclusion and exclusion criteria, 21 articles were analyzed. Most of the studies consider pulse oximetry an alternative method of painless, safe and effective diagnosis, however, the adaptation of an instrument for exclusive dental use is necessary for an exact measurement of the oxygen saturation in the dental pulp. Technological advances in the clinical field of dentistry have led us to search for new clinical diagnostic techniques to improve the care and treatment of patients who come every day to receive a dental consultation. Conclusions: In recent years, pulse oximetry has proven to be an effective diagnostic tool for the diagnosis of pulp vitality. The analysis of the articles included in this review concludes that pulse oximetry is an innovative technique that can be used as an adjunct diagnostic tool in the diagnosis of pulp vitality.

Keywords: Oximetry, dental pulp cavity, pulpitis, diagnosis, dental pulp test.

\footnotetext{
* Alumna de la Maestría en Ciencias Biomédicas.

‡ Docente-Investigador. Unidad Académica de Odontología.

$\S$ Docente-Investigador. Unidad Académica de Enfermería.
}

Universidad Autónoma de Zacatecas «Francisco García Salinas», Zacatecas, México.

Recibido: 31 de diciembre de 2020. Aceptado: 25 de marzo de 2021.

Citar como: de la Rosa-Reyes M, Aguilera-Galaviz LA, Araujo-Espino R, Cepeda-Argüelles Ó, Gaitán-Fonseca C, Bermúdez-Jiménez C. Oximetría de pulso y su eficacia para diagnosticar vitalidad pulpar. Rev ADM. 2021; 78 (2): 84-89. https://dx.doi.org/10.35366/99283 


\section{INTRODUCCIÓN}

$\mathrm{L}$ a vitalidad pulpar es un aspecto clínico evaluado por los profesionales de la salud oral para determinar el suministro de sangre dentro de la pulpa dental, el cual es un indicativo de importancia para dirigir la ruta de tratamiento en el paciente. ${ }^{1,2}$ La vitalidad pulpar está dada por un conjunto íntegro de nervios, arterias y venas que se introducen por el ápice hasta el interior de la cámara pulpar de los órganos dentales. ${ }^{3}$ El dolor es un síntoma que puede no mostrar el verdadero estado del tejido pulpar debido a los diferentes umbrales de dolor y, por otro lado, las pruebas convencionales (palpación y percusión) reflejan el estado sensitivo de la pulpa, mas no su vitalidad al tener limitaciones para determinar un diagnóstico preciso. Además, estas pruebas son molestas para los pacientes, lo que ocasiona distintos contratiempos de cooperación por parte de ellos. ${ }^{4}$

Debido a esto, se han utilizado diferentes métodos experimentales de diagnóstico para evaluar la circulación del tejido pulpar y tratar de obtener una mayor exactitud en el diagnóstico. Estos métodos incluyen técnicas bastante invasivas, como son el uso de aclaramiento de radioisótopos utilizando xenón (Xe) en un modelo experimental animal, ${ }^{5}$ una técnica de desaturación de gas dihidrógeno $\left(\mathrm{H}_{2}\right)$ para medir el flujo de sangre pulpar ${ }^{6,7}$ y medición del flujo sanguíneo y consumo de oxígeno $\left(\mathrm{O}_{2}\right) \cdot{ }^{8}$ Entre las técnicas no invasivas destacan el uso de flujometría mediante láser Doppler, ${ }^{9,10}$ medición de la temperatura de la superficie del diente, ${ }^{11,12}$ espectrofotometría de doble longitud de onda ${ }^{13}$ y la oximetría de pulso. ${ }^{14}$ Esta última es un método ampliamente utilizado en el campo de la medicina. Es un método simple, continuo, no invasivo, para vigilar de manera periférica el porcentaje de hemoglobina $(\mathrm{Hb})$ saturada con oxígeno $\left(\mathrm{O}_{2}\right)$ por el paso de longitudes de onda específicas a través de la sangre $\left(\mathrm{SpO}_{2}\right)$. Este método tiene su principio fundamental en la ley de Beer-Lambert. ${ }^{15}$

La oximetría de pulso parece ser una innovación tecnológica para el diagnóstico endodóntico que puede permitir el análisis del estado de la pulpa durante la intervención odontológica y monitoreo en tiempo real de la vitalidad pulpar. La medición de los niveles de $\mathrm{SpO}_{2}$ en el tejido pulpar puede proveer nuevas perspectivas para diagnósticos más precisos de estado pulpar, lo que sin duda evitaría innecesarias intervenciones endodónticas y proporcionar una base de evidencia para estudios Clínicos adicionales. ${ }^{16}$

El objetivo de la presente revisión es evaluar la eficacia de la oximetría de pulso como método de diagnóstico de la vitalidad pulpar en diferentes estudios reportados en la literatura científica.

\section{METODOLOGÍA}

Se consultaron bases de datos en plataformas digitales para la obtención de material bibliográfico (Google Aca-

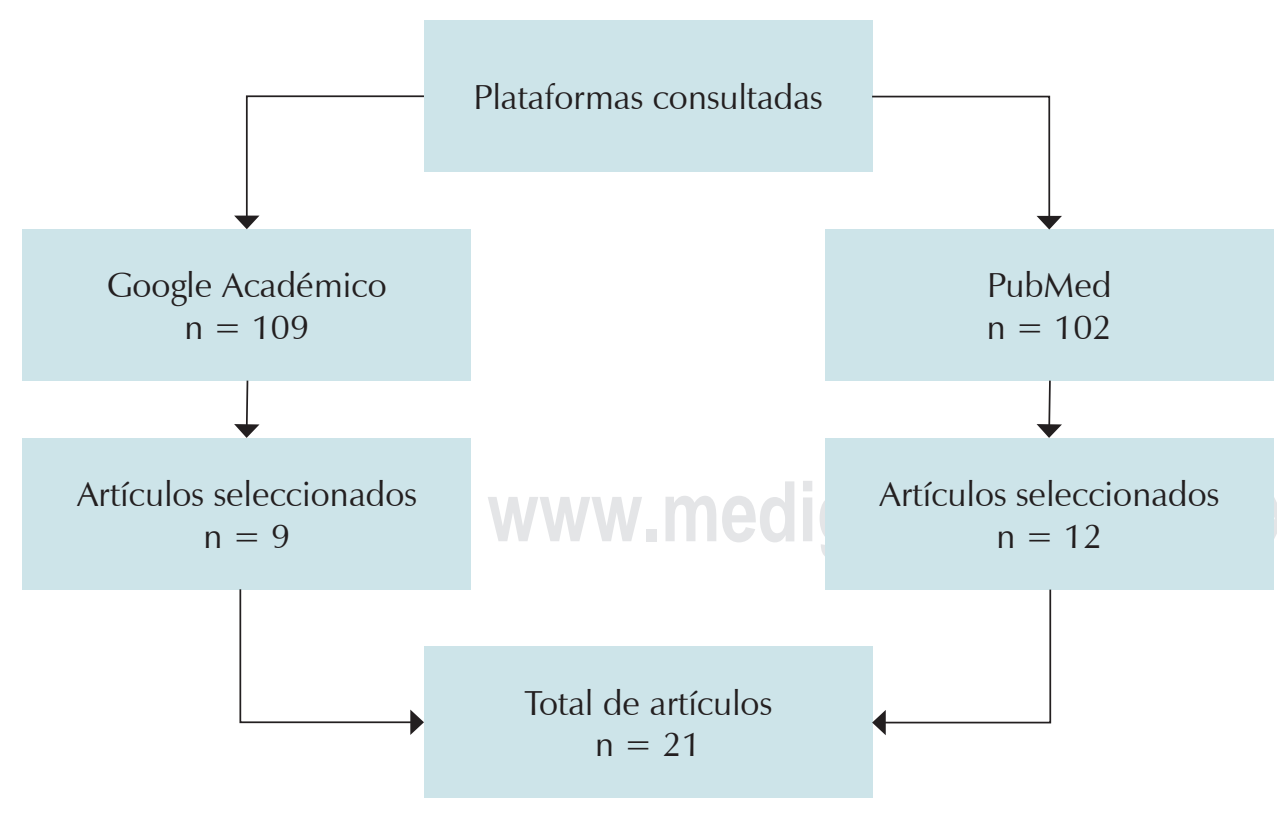

Figura 1:

Diagrama de flujo del número de artículos encontrados y seleccionados. 
Tabla 1: Principales métodos de diagnóstico utilizados para evaluar sensibilidad pulpar.

\begin{tabular}{ll} 
Método de diagnóstico & Descripción \\
\hline Pruebas térmicas & $\begin{array}{l}\text { Se basan en la aplicación de agentes en los dientes. Para aumentar o disminuir la temperatura y para } \\
\text { estimular la pulpa. Las respuestas sensoriales a través de la conducción térmica ha sido la modalidad más } \\
\text { utilizada para pruebas de pulpa }{ }^{18}\end{array}$ \\
Pruebas eléctricas & $\begin{array}{l}\text { Funcionan bajo la premisa de que los estímulos eléctricos causan un cambio iónico a través de la membrana } \\
\text { neural, lo que induce una acción potencial con una rápida acción de salto en los nodos de Ranvier en nervios } \\
\text { mielinizados }\end{array}$ \\
Pruebas de la cavidad & $\begin{array}{l}\text { La preparación de una cavidad de prueba ha sido sugerida como último recurso en un diente donde no hay } \\
\text { otros medios que pueden determinar el estado de la pulpa. }{ }^{20} \text { Consiste en cortar dentina usando una fresa de } \\
\text { alta o baja velocidad sin anestesia local, esto puede dar alguna indicación de si el elemento sensorial de la } \\
\text { pulpa sigue funcionando }\end{array}$
\end{tabular}

Tabla 2: Principales métodos de diagnóstico utilizados para evaluar vitalidad pulpar.

Método de diagnóstico Descripción

Flujometría mediante Esta técnica electroóptica utiliza una fuente láser que es dirigida a la pulpa, y la luz láser viaja a la pulpa láser Doppler

Oximetría de pulso utilizando los túbulos dentinarios como guías ${ }^{22}$

Corresponde a una técnica de monitoreo de la saturación de oxígeno, efectiva y objetiva, ampliamente usada en medicina. Debido a que las unidades comerciales están cada vez más pequeñas y económicas, son aplicables para el diagnóstico endodóntico ${ }^{23}$

démico y PubMed) y se utilizaron las siguientes combinaciones de vocabulario controlado $(\mathrm{MeSH})$ : «Oximetry AND dental pulp test AND diagnosi AND diagnosis». Los criterios para revisión de la literatura se describen a continuación:

\section{Criterios de inclusión}

a. Ensayos clínicos aleatorizados controlados, estudios comparativos, informes de casos y revisiones críticas.

b. Estudios que informan sobre el uso de la oximetría de pulso para determinar la saturación de oxígeno en pulpas dentales.

c. Estudios que involucran grupos experimentales o grupos de control que presentan vitalidad pulpar normal.

d. Estudios que involucran grupos experimentales con vitalidad pulpar alterada causada por procesos inflamatorios o lesiones dentales traumáticas.
2. Criterios de exclusión

a. Estudios que utilicen la oximetría de pulso de manera indirecta.

b. Estudios que evalúen procedimientos de sedación en pacientes pediátricos.

c. Estudios que utilicen la oximetría de pulso en otras disciplinas odontológicas como la periodoncia.

En la Figura 1 se resume el diagrama de flujo para el número de artículos encontrados y seleccionados para esta revisión.

\section{RESULTADOS}

Uno de los mayores desafíos en odontología es el diagnóstico preciso de la vitalidad de la pulpa, ya que las pruebas eléctricas y térmicas convencionales no son totalmente confiables. ${ }^{17}$ Esta técnica aplica un principio conocido como la ley de Beer-Lambert, en la cual se establece que una concentración desconocida de un soluto (por ejemplo, 
la hemoglobina) disuelto en un solvente conocido (sangre) puede evaluarse mediante la absorción de luz del soluto. ${ }^{24}$ Esta oximetría de pulso se mide mediante un aparato en el cual la luz pasa de un diodo fotoeléctrico a través de una parte del cuerpo (por ejemplo, el esmalte, dentina y pulpa) hacia un receptor. La diferencia entre la luz emitida y la luz recibida se calcula en un microprocesador para proporcionar lecturas de latidos por minuto (bpm: beats per minute) y saturación de oxígeno $\left(\mathrm{SpO}_{2}\right){ }^{2}{ }^{2}$ El diodo emite dos longitudes de onda de luz para detectar la hemoglobina oxigenada (sangre arterial) y la hemoglobina desoxigenada (sangre venosa). Este resultado es la relación de la absorbancia de las longitudes de onda que proporciona el porcentaje de oxigenación de la sangre (de 95 a $100 \%$ en condiciones normales). ${ }^{25}$ Este signo indica que por la vascularización que existe en esta área anatómica y mediante el transporte de hemoglobina se pueden obtener datos de la oxigenación del tejido más precisos, y no únicamente basarnos en los datos referidos por el paciente. ${ }^{26}$ En el caso de las pruebas de vitalidad pulpar más utilizadas, se realiza una evaluación del suministro de sangre de la pulpa, por lo que el tejido pulpar puede tener un suministro vascular adecuado, pero no necesariamente inervado, en consecuencia, estas pruebas pueden arrojar falsos positivos o falsos negativos. ${ }^{27}$

Anderson, Anderson y Glanze mencionan que la sensibilidad se define como la capacidad de responder a un estímulo, ${ }^{28}$ por lo tanto, la oximetría de pulso pudiera ser un método preciso y apropiado para evaluar las pruebas de vitalidad pulpar, incluso aún más que las pruebas clínicas típicas y comunes, como lo son las pruebas térmicas y eléctricas, dado que estas últimas no detectan o miden el suministro de oxígeno en la sangre de la pulpa dental. ${ }^{21}$
Los principales métodos de diagnóstico del estado pulpar se enfocan en la sensibilidad pulpar (Tabla 1), así como en la detección de la vitalidad pulpar (Tabla 2). Al monitorear los cambios en la saturación de oxígeno la oximetría de pulso puede detectar inflamación de la pulpa o necrosis parcial en dientes que aún responden de manera sensible a otras pruebas. ${ }^{23}$ En la Tabla 3 se resumen los hallazgos más importantes encontrados en la literatura revisada.

\section{DISCUSIÓN}

Dentro de los resultados mencionados por los autores en los diversos estudios realizados con la oximetría de pulso se determina lo siguiente: los valores de saturación de oxígeno obtenidos en los dientes son, por lo general, más bajos que los obtenidos en el dedo del paciente. ${ }^{29}$ Esta observación puede tener dos explicaciones: a) se da por el aislamiento de la pulpa por los tejidos duros circundantes que son un obstáculo para la detección de la vascularización; ${ }^{3}$ b) se puede dar por la difracción del infrarrojo de la luz por el prisma del esmalte y la dentina, esto puede dar lugar a una disminución de lecturas de $\mathrm{SpO}_{2} \cdot{ }^{29,31} \mathrm{En}$ los resultados de algunos estudios se sugiere que las lecturas de $\mathrm{SpO}_{2}$ por debajo del $70 \%$ puede ser considerado como una respuesta positiva en la evaluación de flujo sanguíneo pulpar. ${ }^{4}$

A pesar de que Gopikrishna y colaboradores demostraron con anterioridad que las pruebas de oxímetro de pulso tienen una menor especificidad en comparación con las pruebas frías y eléctricas, ${ }^{3}$ la aceptación por parte de los pacientes del oxímetro de pulso es alta, ya que, al tener una naturaleza no invasiva, puede conducir a una mayor aceptación y cooperación por parte de éstos,

Tabla 3: Hallazgos más importantes sobre la oximetría de pulso en la literatura revisada.

Variable

Valores de saturación

de oxígeno

Lecturas de $\mathrm{SpO}_{2}$

Especificidad

Aceptación por parte

de los pacientes

Dientes permanentes
Descripción

Los valores obtenidos en los dientes son generalmente más bajos que los obtenidos en el dedo del paciente

Las lecturas de $\mathrm{SpO}_{2}$ por debajo del $70 \%$ pueden ser consideradas como una respuesta positiva en la evaluación del flujo sanguíneo pulpar

Las pruebas de oxímetro de pulso tienen una menor especificidad en comparación con pruebas frías y eléctricas

La aceptación por parte de los pacientes del oxímetro de pulso es alta, ya que, al tener una naturaleza no invasiva, no se presenta dolor alguno al momento de la prueba

Análisis demostraron que la media de saturación de oxígeno en pulpas dentales normales de dientes permanentes fue superior a $87 \%$
Autores, año

Goho et al., $1999^{29}$

Pozzobon et al., 2011

Gopikrishna et al., $2006^{3}$

Pozzobon et al., 2011

Bruno et al., 2014 30 
inclusive en pacientes pediátricos, durante los procedimientos de diagnóstico pulpar, ${ }^{4}$ lo cual podría representar una ventaja en pacientes no cooperadores.

Los factores relacionados con la edad, como la disminución del volumen de la pulpa causada por la deposición de tejido mineralizado, pueden resultar en lecturas menos efectivas o falsos negativos con la oximetría de pulso, ${ }^{3} \mathrm{O}$ bien un aumento del grosor de la dentina. ${ }^{4}$ Los análisis demostraron que la media de saturación de oxígeno en pulpas dentales normales de dientes permanentes fue superior a $87 \%$. Este valor podría utilizarse como punto de referencia para dientes permanentes mediante el uso de oxímetros de pulso para diagnosticar la vitalidad pulpar. ${ }^{30}$

\section{CONCLUSIONES}

El único inconveniente que se ha encontrado en el método de la oximetría de pulso aplicado al diagnóstico del estado pulpar es que no existe hasta el momento un oxímetro de pulso exclusivo de uso odontológico y que pueda ser utilizado como un test de vitalidad confiable, por lo cual quienes recurran a este método diagnóstico tendrán que enfrentarse a la realización de una adaptación técnica que podría arrojar datos erróneos si no se realizan las adecuaciones correctas; sin embargo, en la mayoría de los artículos analizados en esta revisión, la oximetría de pulso resultó ser una herramienta confiable, segura, eficaz y bien aceptada como un adyuvante en el diagnóstico de la vitalidad pulpar.

\section{REFERENCIAS}

1. Baumgardner K, Walton R, Osborne J, Born J. Induced hypoxia in rat pulp and periapex demonstrated by $3 \mathrm{H}$-misonidazole retention. J Dent Res. 1996; 75 (10): 1753-1760. doi: 10.1177/00220345960750100801.

2. Trowbridge HO, Kim S. Pulp development, structure and function. In: Cohen S, Burns RC, eds. Pathways of the Pulp. St. Louis: Mosby, 1998: 386-424.

3. Gopikrishna V, Tinagupta K, Kandaswamy D. Evaluation of efficacy of a new custom-made pulse oximeter dental probe in comparison with the electrical and thermal tests for assessing pulp vitality. J Endod. 2007; 33 (4): 411-414. doi: 10.1016/j. joen.2006.12.003.

4. Pozzobon MH, De Sousa VR, Hecke AM, Reyes-Carmona J, Teixeira CS, De Souza BD et al. Assessment of pulp blood flow in primary and permanent teeth using pulse oximetry. Dental Traumatol. 2011; 27 (3): 184-188. doi: 10.1111/j.1600-9657.2011.00976.x.

5. Kim S, Schuessler G, Chien S. Measurement of blood flow in the dental pulp of dogs with the 133xenon washout method. Arch Oral Biol. 1983; 28 (6): 501-505. doi: 10.1016/0003-9969(83)90181-4.

6. Tonder $\mathrm{KH}$, Aukland K. Blood flow in the dental pulp in dogs measured by local $\mathrm{H} 2$ gas desaturation technique. Arch Oral Biol. 1975; 20 (1): 73-79. doi: 10.1016/0003-9969(75)90155-7.

7. Meyer MW, Path MG. Blood flow in the dental pulp of dogs determined by hydrogen polarography and radioactive microsphere methods. Arch Oral Biol. 1979; 24 (8): 601-605. doi: 10.1016/0003-9969(79)90020-7.

8. De Leon RL, Path MG, Meyer MW. Blood flow and oxygen consumption in steroid-treated dental pulps. Oral Surg Oral Med Oral Pathol. 1978; 45 (5): 784-788. doi: 0.1016/00304220(78)90155-X.

9. Ghouth N, Duggal MS, Kang J, Nazzal H. A diagnostic accuracy study of laser Doppler flowmetry for the assessment of pulpal status in children's permanent incisor teeth. J Endod. 2019; 45 (5): 543548. doi: 10.1016/j.joen.2019.01.017.

10. Ghouth N, Duggal MS, BaniHani A, Nazzal H. The diagnostic accuracy of laser Doppler flowmetry in assessing pulp blood flow in permanent teeth: a systematic review. Dental Traumatology. 2018; 34 (5): 311-319. doi: 10.1111/edt.12424.

11. Smith E, Dickson M, Evans AL, Smith D, Murray CA. An evaluation of the use of tooth temperature to assess human pulp vitality. Int Endod J. 2004; 37 (6): 374-380.

12. Jafarzadeh $\mathrm{H}$, Udoye $\mathrm{Cl}$, Kinoshita J-I. The application of tooth temperature measurement in endodontic diagnosis: a review. J Endod. 2008; 34 (12): 1435-1440. doi: 10.1111/j.13652591.2004.00815.x.

13. Nissan R, Trope M, Zhang C-D, Chance B. Dual wavelength spectrophotometry as a diagnostic test of the pulp chamber contents. Oral Surg Oral Med Oral Pathol. 1992; 74 (4): 508-514. doi: 10.1016/0030-4220(92)90304-9.

14. Radhakrishnan S, Munshi AK, Hegde AM. Pulse oximetry: a diagnostic instrument in pulpal vitality testing. J Clin Pediatr Dent. 2002; 26 (2): 141-145. doi: 0.17796/ jcpd.26.2.2j25008jg6u86236.

15. López-Herranz GP. Oximetría de pulso: a la vanguardia en la monitorización no invasiva de la oxigenación. Rev Med Hosp Gen Mex. 2003; 66 (3): 160-169.

16. Lima LF, ALENCAR AHGd, Decurcio DdA, Silva JA, Favarao IN, Loureiro MAZ et al. Effect of dental bleaching on pulp oxygen saturation in maxillary central incisors-a randomized clinical trial. J Appl Oral Sci. 2019; 27: e20180442. doi: 10.1590/1678-77572018-0442.

17. Dastmalchi N, Jafarzadeh $H$, Moradi S. Comparison of the efficacy of a custom-made pulse oximeter probe with digital electric pulp tester, cold spray, and rubber cup for assessing pulp vitality. J Endod. 2012; 38 (9): 1182-1186. doi: 10.1016/j. joen.2012.06.012.

18. Ehrmann E. Pulp testers and pulp testing with particular reference to the use of dry ice. Aust Dent J. 1977; 22 (4): 272-279.

19. Bender I. Reversible and irreversible painful pulpitides: diagnosis and treatment. Aust Endod. 2000; 26 (1): 10-14. doi: 10.1111/ j.1747-4477.2000.tb00144.x.

20. Rowe A, Ford TP. The assessment of pulpal vitality. Int Endod J. 1990; 23 (2): 77-83. doi: 10.1111/j.1365-2591.1990. tb00843.x.

21. Chen E, Abbott PV. Dental pulp testing: a review. International journal of Dentistry. 2009; 2009: 365785. doi: $10.1155 / 2009 / 365785$

22. Matthews B, Vongsavan N. Advantages and limitations of laser Doppler flow meters. International endodontic journal. 1993; 26 (1): 9-9. doi: 0.1111/j.1365-2591.1993.tb00531.x.

23. Jafarzadeh $\mathrm{H}$, Rosenberg PA. Pulse oximetry: review of a potential aid in endodontic diagnosis. J Endod. 2009; 35 (3): 329-333. doi: 10.1016/j.joen.2008.12.006.

24. Rajkumar A, Karmarkar A, Knott J. Pulse oximetry: an overview. J Perioper Practic. 2006; 16 (10): 502-504. doi: 10.1177/175045890601601005. 
25. Alexander CM, Teller LE, Gross JB. Principles of pulse oximetry: theoretical and practical considerations. Anesth Analg. 1989; 68 (3): 368-376.

26. Schnettler JM, Wallace JA. Pulse oximetry as a diagnostic tool of pulpal vitality. J Endod. 1991; 17 (10): 488-490. doi: 0.1016/ S0099-2399(06)81795-4.

27. Johnson J. Evaluation of teeth vitality after subapical osteotomy. J Oral Surg. 1969; 27: 256-257.

28. Anderson NK AL, Glanze DW. Mosby's medical, nursing, and allied health dictionary. 6th ed. St. Louis, Miss, USA: Mosby; 2002.

29. Goho C. Pulse oximetry evaluation of vitality in primary and immature permanent teeth. Pediatr Dent. 1999; 21: 125-127.

30. Bruno KF, Barletta FB, Felippe WT, Silva JA, de Alencar AHG, Estrela C. Oxygen saturation in the dental pulp of permanent teeth: a critical review. J Endod. 2014; 40 (8): 1054-1057. doi: 10.1016/j. joen.2014.04.011.
31. Munshi A, Hegde A, Radhakrishnan S. Pulse oximetry: a diagnostic instrument in pulpal vitality testing. J Clin Pediatr Dent. 2003; 26 (2): 141-145. doi: 10.17796/ jcpd.26.2.2j25008jg6u86236.

Correspondencia:

Carlos Bermúdez-Jiménez

E-mail: carlosber8@uaz.edu.mx

carlosbj8@gmail.com

Conflicto de intereses: El autor declara no tener ningún conflicto de intereses.

Aspectos éticos: Ninguno.

Financiamiento: Ninguno. 